\title{
Is dynamic heterogeneity of water in presence of a protein denaturing agent different from that in presence of a protein stabilizer? A molecular dynamics simulation study
}

\author{
SANDIPA INDRA and RANJIT BISWAS* \\ Chemical, Biological and Macromolecular Sciences, S. N. Bose National Centre for Basic Sciences, \\ Block JD, Sector III, Salt Lake, Kolkata 700 098, India \\ e-mail: ranjit@bose.res.in
}

MS received 9 July 2016; revised 12 September 2016; accepted 18 October 2016

\begin{abstract}
Rotational and translational dynamic heterogeneities (DHs) of ambient aqueous solutions of trimethylamine-N-oxide (TMAO) and tetramethylurea (TMU) at several solute concentrations have been investigated and compared. Motional characteristics of water molecules at solute interfaces and in bulk solutions have been thoroughly examined for the search of slow dynamics. Note, TMAO possesses zwitterionic structure and is a protein stabilizer whereas TMU is a neutral dipolar molecule and a strong denaturant. Results suggest that water-TMAO solutions possess stronger DH than water-TMU solutions with the solute concentration dependence being stronger for TMAO than for TMU. Diffusive dynamics slows down near the solute surface for both the solutes. Solvation structure shows TMAO-water interaction is stronger than TMU-water interaction, producing longer H-bond fluctuation timescale in TMAO solutions. In short, this paper presents, for the first time, a systematic and comparative study of motional features and inter-species interactions between aqueous solutions containing solutes that differ in their individual impacts on protein stability.
\end{abstract}

Keywords. Dynamic heterogeneity; aqueous solutions; protein stabilizer; denaturant; computer simulations

\section{Introduction}

Trimethylamine-N-oxide (TMAO) is an amphiphile that can protect the volume of a living cell by preventing damage from external osmotic or hydrostatic pressure. $^{1,2}$ It has been found that TMAO is a better protein stabilizer than other osmolytes such as betaine, methylamine, etc. ${ }^{3,4}$ However, whether TMAO affects protein stability by directly interacting with $\operatorname{protein}^{5,6}$ or via modification of water interaction and dynamics ${ }^{7,8}$ is still under debate. Tetramethylurea (TMU), another amphiphilic molecule, acts as a protein destabilizer, and is a stronger denaturant than urea. ${ }^{5,6}$ Interestingly, these two molecules are of similar size $\left(r_{T M A O} \sim 3 \AA\right.$ and $r_{T M U} \sim 3.5 \AA, r$ being van der Waals radius $)^{9,10}$ although TMAO possesses a zwitterion structure while TMU is a dipolar amphiphile (Scheme 1). Because of this difference in chemical nature, TMAO is a solid at room temperature whereas TMU is a liquid. TMAO contains three hydrophobic methyl groups, and a negatively charged oxygen atom which can accept more than two hydrogen bonds (H-bonds). TMU consists of four methyl groups, and a carbonyl oxygen which can form, on an average, H-bonds with two water molecules. ${ }^{11}$

\footnotetext{
*For correspondence
}

These contrasting scenarios have motivated the present study, as in several previous works,,${ }^{11-25}$ where immobilization or extreme slowing down of water molecules at the interfaces of these two chemically different amphiphiles has been investigated and compared via molecular dynamics simulations.

This area has witnessed a resurgence in the last several years as a series of femtosecond midinfrared measurements ${ }^{12-17}$ investigated the reorientational dynamics of interfacial water molecules in aqueous solutions of TMAO and TMU and revisited the iceberg model. ${ }^{26}$ These measurements indicated extreme slowing down of water molecules that surround these solutes and this effective immobilization is independent of amphiphile concentration. These experiments reported, depending on amphiphile identity, a slowing down of $\sim 3-8$ times for the interfacial water molecules over those in the bulk. However, effects of solute clustering on water dynamics were not examined in these studies although TMU aggregates at very low concentrations ${ }^{27}$ whereas TMAO remains unaggregated even at large concentrations. ${ }^{28}$ Interestingly, subsequent ultrafast optical Kerr Effect measurements ${ }^{11}$ indicated a much weaker slowing down (approximately a factor of 2) of the interfacial water molecules with no appreciable changes in the water structure. These 


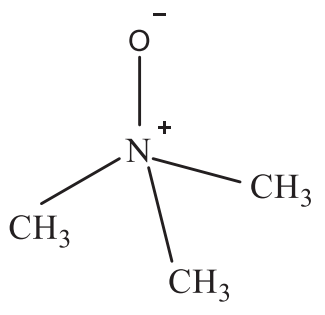

(A)<smiles>CN(C)C(=O)N(C)C</smiles>

(B)
Scheme 1. Chemical structures of (A) Trimethylamine-Noxide (TMAO) and (B) Tetramethylurea (TMU).

Kerr effect spectroscopic results were supported by previous nuclear magnetic resonance (NMR) studies ${ }^{29}$ and molecular dynamics simulations of jump reorientations of interfacial water molecules. ${ }^{19,20}$ These simulation studies ${ }^{19,20}$ also reported no significant decoupling between water translation and reorientation dynamics, and suggested that the slow-down of water dynamics in such solutions occur primarily due to the confining effects at large amphiphile density. In several recent studies, ${ }^{23,30-32}$ slowing down of dynamics of the hydrating water molecules has been explained in terms of reduced rate of switching of $\mathrm{H}$-bond partners near hydrophobic groups, ${ }^{31}$ while another group has indicated that the slow-down in water/TMAO solutions arises from a strong H-bonding between water hydrogen atom and the oxygen atom of TMAO. ${ }^{23}$ It is also argued that proper modelling of the negatively charged oxygen atom is critical for such an observation. However, a systematic comparative study of dynamical heterogeneity $(\mathrm{DH})$ of these solutions has not been made yet.

In this work, we have made a comparative study of water dynamics in ambient aqueous solutions of TMAO and TMU at various concentrations. The difference in interactions between water/TMAO and water/TMU has been explored. DH in terms of non-Gaussian (NG) and new non-Gaussian (NNG) parameters ${ }^{33,34}$ have been calculated both for the interfacial and bulk water molecules at different $X_{\text {Solute }}$. Simulated concentration dependent rotational and translational NG parameters ${ }^{35,36}$ reveal concentration and solute dependences, indicating effects of these solutes on water structure. Computed single particle displacement distributions for water molecules are strongly non-Gaussian for both the solutes at all the concentrations considered. The ratio between the simulated rotational and translational diffusion coefficients of water $\left(D_{R} / D_{T}\right)$ at various concentrations deviates strongly from the hydrodynamics ${ }^{37}$ for both TMAO and TMU. A very weak slow-down of translational diffusive dynamics of interfacial water has been observed. The H-bond fluctuating timescales, ${ }^{38-40}$ lengthen with solute concentration, also show strong solute dependency.

\section{Computational Details}

\subsection{Force Field Description}

We used SPC/E model of water ${ }^{41}$ and specific force fields for TMAO ${ }^{18,28}$ and TMU. ${ }^{24}$ Detailed description of force field and model for TMU has been provided in literature. ${ }^{24}$ In rigid model of TMAO, interaction of different atomic sites of two different molecules is given by,

$U_{i j}\left(r_{i j}\right)=\sum_{i<j}\left\{4 \varepsilon_{i j}\left[\left(\frac{\sigma_{i j}}{r_{i j}}\right)^{12}-\left(\frac{\sigma_{i j}}{r_{i j}}\right)^{6}\right]+\frac{q_{i} q_{j}}{4 \pi \varepsilon_{0} r_{i j}}\right\}$.

Here, the nonbonded interactions are included by Lennard-Jones (LJ) potential and Coulomb interaction. $\varepsilon, \sigma, r$ are the potential well depth, van der Waals

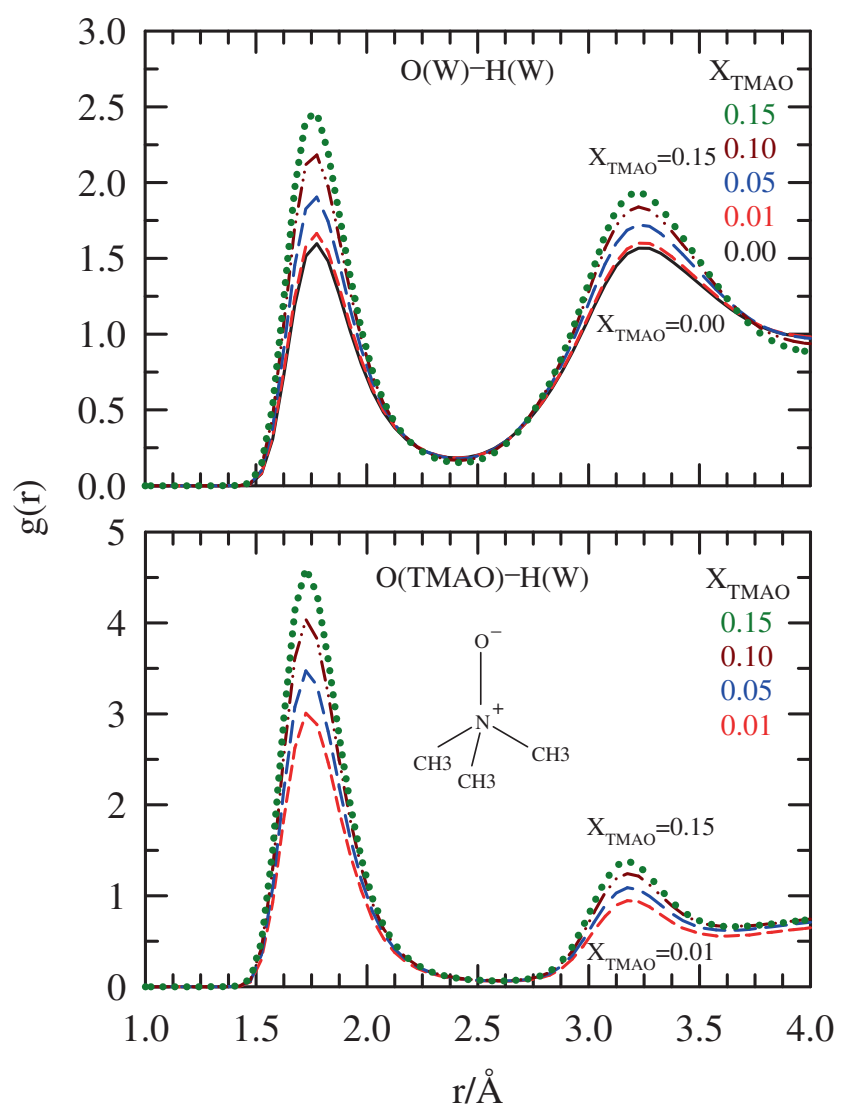

Figure 1. Site-site radial distribution functions, $g(r)$, between different atomic pairs as a function of distance, $r$, at various TMAO mole fractions, $\mathrm{X}_{\mathrm{TMAO}}$. Color codes and line styles are as follows: black solid: pure water, red short-dash: 0.01 , blue medium-dash: 0.05 , dark red dash-dot-dot: 0.10 , dark green dot: 0.15 . Chemical structure of TMAO is also shown as inset of the lower panel. 
radius, and distance between atoms, respectively. The parameter $q$ represents the partial charge of the atom and $\varepsilon_{0}$ the static dielectric constant. LJ interactions between unlike atoms are calculated by the LorentzBerthelot combining rule. ${ }^{42}$

\subsection{Simulation Protocols}

A total of 512 (solute+solvent) molecules in a cubic box with periodic boundary condition were considered. The initial configuration was generated by Packmol. ${ }^{43}$ Simulation was run first using the NPT ensemble at ambient condition and employing the DL_POLY_ Classic ${ }^{44}$ with the Hoover thermostat and barostat relaxation times of $2 \mathrm{ps}$ and $0.5 \mathrm{ps}$, respectively. A $2 \mathrm{~ns}$ simulation in this ensemble was necessary to produce the experimental density. ${ }^{45,46}$ The final configuration obtained via this NPT simulation was then used as the initial configuration for NVT ensemble at the ambient condition using the Nose-Hoover ${ }^{47,48}$ thermostat with relaxation time of $0.5 \mathrm{ps}$. Then, a 5 ns simulation run was completed in which first $1 \mathrm{~ns}$ run was considered for further equilibration in the NVT ensemble, and

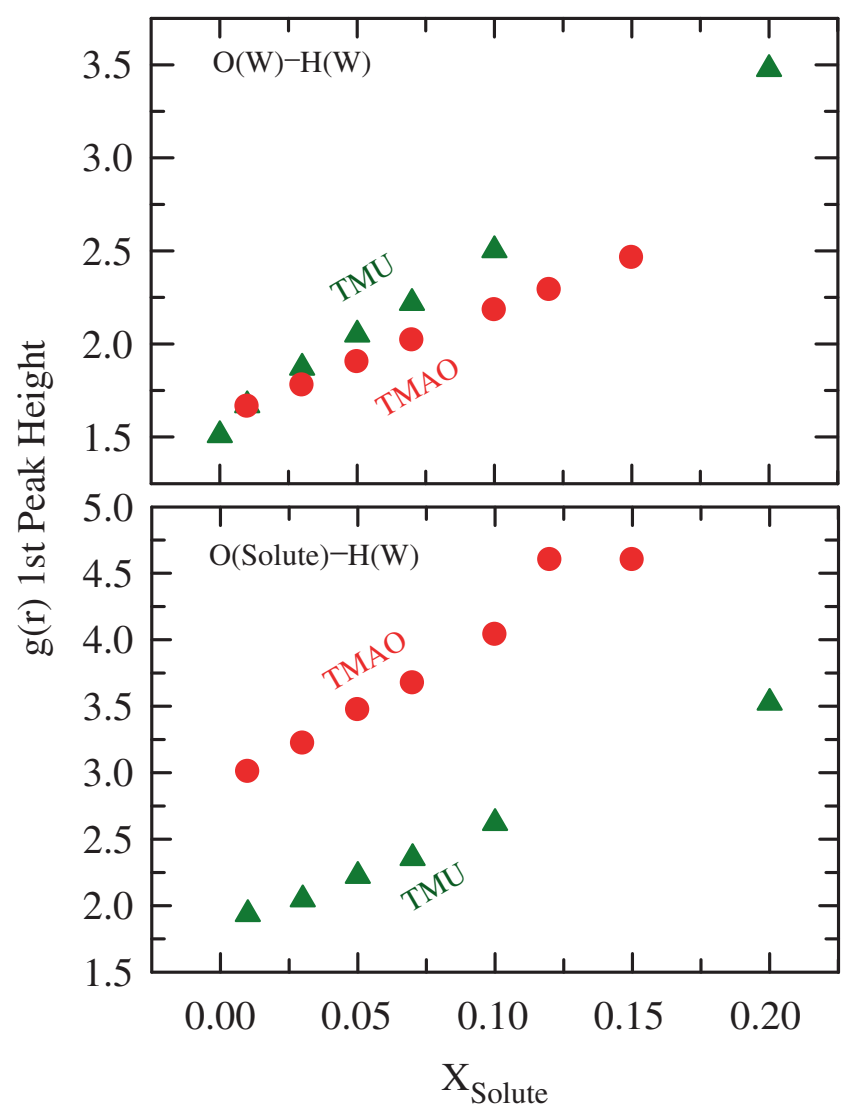

Figure 2. Height of the first peak of simulated $g(r)$ as a function of $X_{\text {Solute }}$. Red solid circles and dark green solid triangles represent (TMAO/water) and (TMU/water) mixtures, respectively. the last $4 \mathrm{~ns}$ run was analyzed for results. Equations of motion were solved by Leapfrog-Verlet algorithm ${ }^{42}$ with time step of $1 \mathrm{fs}$. Verlet neighbour list shell width was set to $1.2 \AA$. Trajectories were saved after each $10 \mathrm{fs}$. Cut off radius was set nearly to the half of the box length. Statistics data were saved after each 5 time steps. The SHAKE algorithm ${ }^{49}$ was employed to constrain all the bonds involving hydrogen atoms. Ewald summation technique ${ }^{42}$ was used for treating the electrostatic interactions.

\section{Results and Discussion}

\subsection{Solvation Structure in the Binary Mixture}

In both the TMAO/water and TMU/water systems, water hydrogen atoms, $\mathrm{H}(\mathrm{W})$ can form $\mathrm{H}$-bonds with the oxygen atoms of TMAO and TMU, O(TMAO) and $\mathrm{O}(\mathrm{TMU})$, respectively. Figure 1 represents the effect of gradual increase of TMAO mole fraction, $X_{T M A O}$, into water on the radial distribution functions
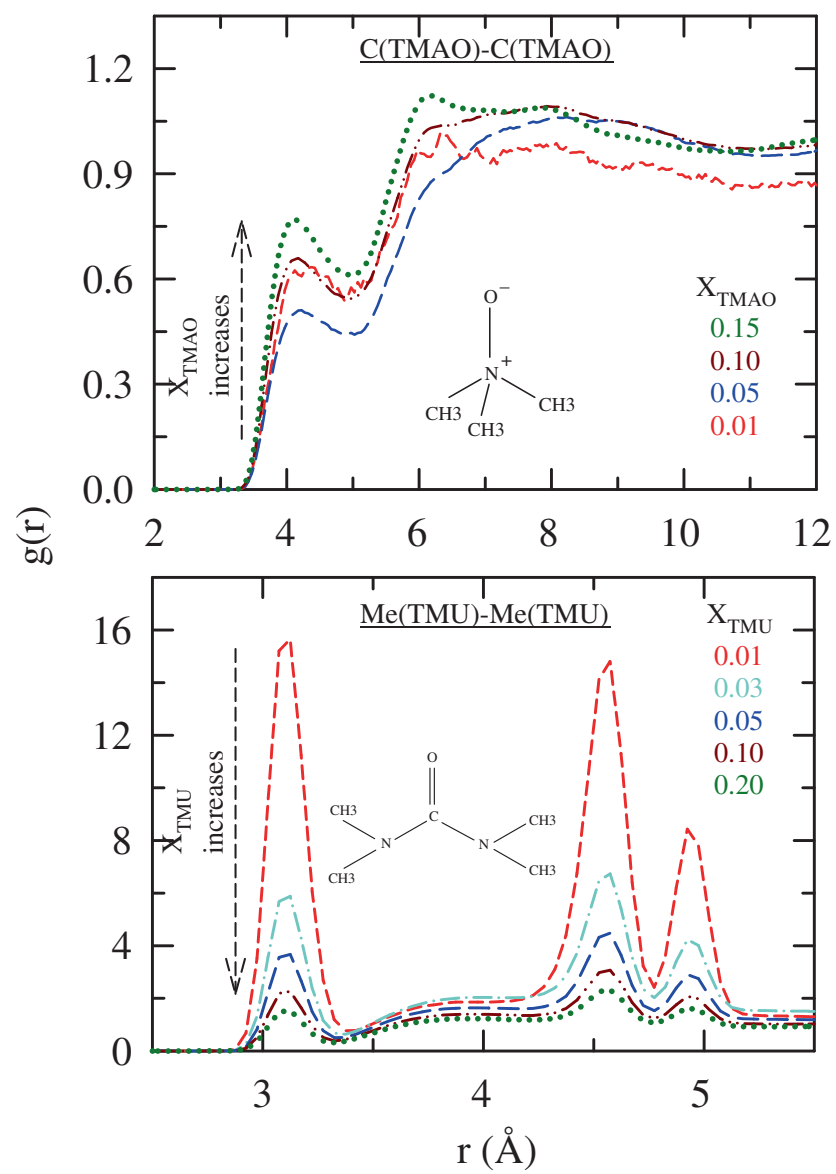

Figure 3. Site-site RDFs between atomic pairs involved in hydrophobic interaction at various concentrations of TMAO and TMU into water as a function of distance, $r$. Representations are color-coded. 
(RDFs) involving $\mathrm{O}(\mathrm{W})-\mathrm{H}(\mathrm{W})$ (upper panel) and $\mathrm{O}(\mathrm{TMAO})-\mathrm{H}(\mathrm{W})$ (lower panel) pairs as a function of distance, $r$. Clearly, addition of TMAO increases $\mathrm{g}(\mathrm{r})$ peak intensities for both the $\mathrm{O}(\mathrm{W})-\mathrm{H}(\mathrm{W})$ and $\mathrm{O}(\mathrm{TMAO})-\mathrm{H}(\mathrm{W})$ distributions, keeping the peak positions unchanged. This suggests that the H-bonding interactions between water-water and water-TMAO increases upon addition of TMAO into water. Similar behaviour was also observed earlier for $\mathrm{g}(\mathrm{r})$ describing $\mathrm{O}(\mathrm{W})-\mathrm{H}(\mathrm{W})$ and $\mathrm{O}(\mathrm{TMU})-\mathrm{H}(\mathrm{W})$ RDFs in TMU/water mixtures. ${ }^{24}$ Note also that the $\mathrm{g}(\mathrm{r})$ peak intensity of $\mathrm{O}(\mathrm{TMAO})-\mathrm{H}(\mathrm{W})$ is uniformly higher $(\sim 80 \%)$ at each $X_{T M A O}$ than the corresponding $\mathrm{O}(\mathrm{W})-\mathrm{H}(\mathrm{W}) \mathrm{RDF}$. This is probably due to the enhanced electrostatic interaction because of the negative charge on the oxygen atom in TMAO. This was not found for TMU/water systems. ${ }^{24}$ Figure S1 (Supplementary Information) presents the RDFs corresponding to the interaction between oxygen atoms of water-water, water-solute and solute-solute for both the TMAO/water and TMU/water systems. We found that for the same concentration, $\mathrm{O}(\mathrm{W})-\mathrm{O}(\mathrm{W})$ $\mathrm{g}(\mathrm{r})$ peak intensity is slightly lower in the binary mixture of TMAO/water than in that of TMU/water. This effect is opposite for $\mathrm{O}(\mathrm{W})-\mathrm{O}$ (solute) interaction for both the systems. This is expected because the enhanced electrostatic property of TMAO molecules drives them to preferentially interact with water.

Figure 2 shows the first peak heights of $\mathrm{O}(\mathrm{W})-\mathrm{H}(\mathrm{W})$ and $\mathrm{O}$ (solute) $-\mathrm{H}(\mathrm{W})$ RDFs as a function of $X_{\text {Solute }}$ for both the TMAO/water and TMU/water systems. Clearly, water-water $\mathrm{H}$-bond coordination number is more in TMU/water system, whereas TMAO/water system supports greater water-solute H-bond coordination number. This is expected because of the inherent difference in charge distributions for these solutes.

RDFs describing interaction among methyl groups of solute molecules in these aqueous solutions are shown in Figure 3. Note that for aqueous solutions of TMAO, Me-Me $\mathrm{g}(\mathrm{r})$ grows slowly with $X_{T M A O}$ (higher $\mathrm{g}(\mathrm{r})$ peak intensity at $X_{T M A O}=0.01$ indicates nothing but poor averaging). For TMU solutions, in contrast, the corresponding RDF shows intense peak at $X_{T M U}=0.01$ and then falls of abruptly upon increasing $X_{T M U}=0.03$. This is a reflection of TMU aggregation at very low concentration. ${ }^{21,27}$ All these suggest that TMAO molecules possess higher propensity to interact with water than among themselves. This is further confirmed from N(TMAO)-O(TMAO) RDF shown in
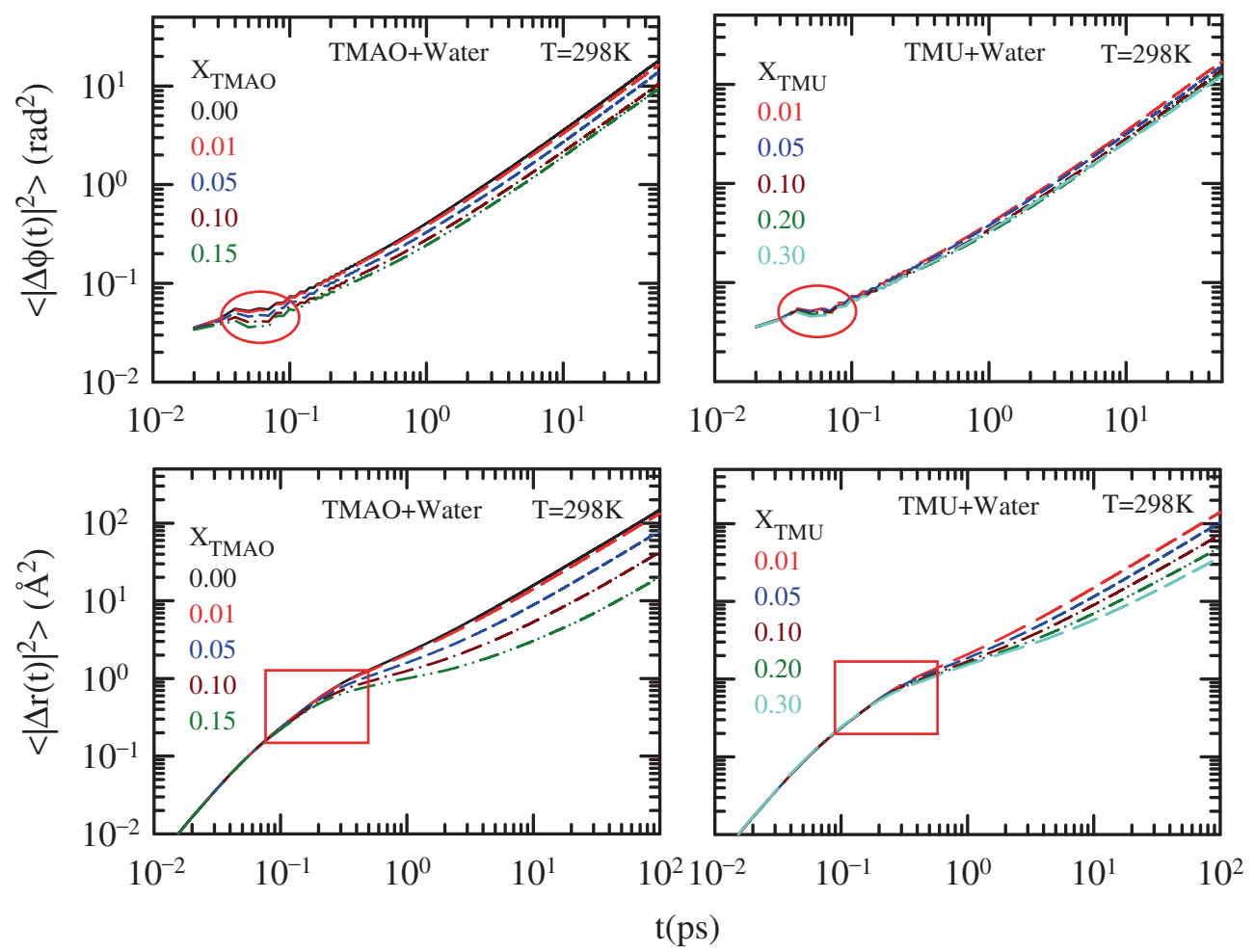

Figure 4. Plot of rotational mean squared displacement (RMSD), $\left\langle\left|\Delta \vec{\phi}_{i}(t)\right|^{2}\right\rangle$ of water -OH bonds and translational mean squared displacement (MSD), $\left\langle|\Delta \vec{r}(t)|^{2}\right\rangle$ of water at different concentrations of TMAO (left panels) and TMU (right panels) with time, $t$. Circles in $\left\langle\left|\Delta \vec{\phi}_{i}(t)\right|^{2}\right\rangle$ plots and rectangles in $\left\langle|\Delta \vec{r}(t)|^{2}\right\rangle$ plots are drawn to highlight the different behaviours of RMSD and MSD at the time of transition from inertial to diffusive regimes. Representations are color-coded. 
Figure S2 (Supplementary Information). TMU also shows a similar trend but to a lesser extent than TMAO.

\subsection{Rotational and Translational Diffusions of Water}

Rotational diffusion coefficient, $D_{R}$, and translational diffusion coefficient, $D_{T}$, for water molecules in these aqueous binary mixtures have been calculated from the slope of rotational mean squared displacement (RMSD) and translational mean squared displacement (MSD) respectively by using the following equations: ${ }^{35-37,50}$

$$
\begin{aligned}
D_{R} & =\left[\frac{1}{4 t}\left(\left\langle\left|\Delta \vec{\phi}_{i}(t)\right|^{2}\right\rangle\right)\right]_{t \rightarrow \infty} \\
& =\left[\frac{1}{4 t}\left\{\frac{1}{N}\left\langle\sum_{i=1}^{N}\left|\vec{\phi}_{i}(t)-\vec{\phi}_{i}(0)\right|^{2}\right\rangle\right\}\right]_{t \rightarrow \infty}
\end{aligned}
$$
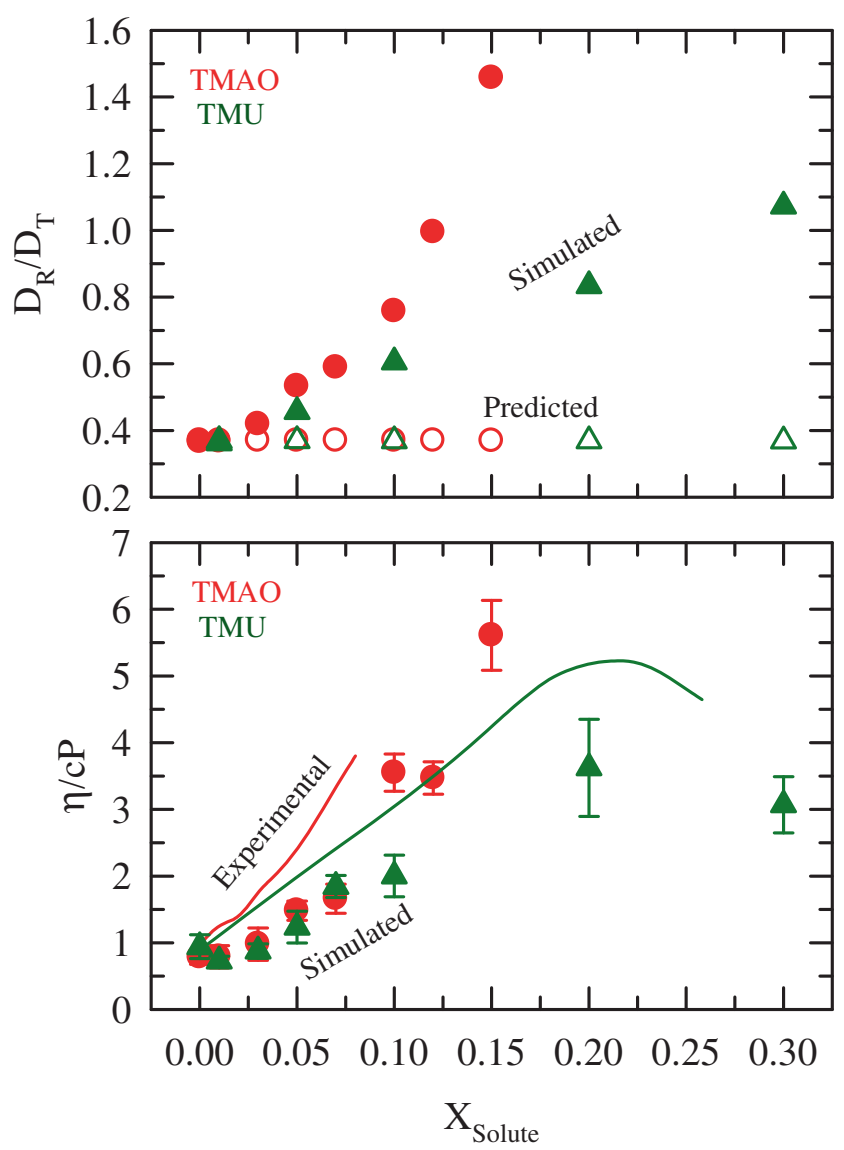

Figure 5. $X_{\text {Solute }}$ dependence of the ratio between the rotational and translational diffusion coefficients, $D_{R} / D_{T}$ (upper panel), and viscosity coefficients, $\eta$ (lower panel), for aqueous solutions of TMAO and TMU. Stick hydrodynamic predictions (upper panel) and measured viscosity coefficients $(\eta)$ for these solutions (lower panel) are also shown. Experimental ${ }^{45,51}$ and simulated $\eta$ are represented by the lines and symbols, respectively. Red represents TMAO and dark green TMU. Note the ratio, $D_{R} / D_{T}$, is scaled by $\left(\AA^{-1}\right)^{2}$. where, $\vec{\phi}_{i}(t)-\phi_{i}(0)=\int_{0}^{t} d t^{\prime} \vec{\omega}_{i}\left(t^{\prime}\right),\left\langle\left|\Delta \vec{\phi}_{i}(t)\right|^{2}\right\rangle$ and $\vec{\omega}_{i}(t)$ represent the RMSD and the angular velocity of $-\mathrm{OH}$ bond vector of water respectively. $N$ is the total number of molecules and $t$ is time.

$$
\begin{aligned}
D_{T} & =\left[\frac{1}{6 t}\left(\left\langle|\Delta \vec{r}(t)|^{2}\right\rangle\right)\right]_{t \rightarrow \infty} \\
& =\left[\frac{1}{6 t}\left\{\frac{1}{N}\left\langle\sum_{i=1}^{N}\left|\vec{r}_{i}(t)-\vec{r}_{i}(0)\right|^{2}\right\rangle\right\}\right]_{t \rightarrow \infty}
\end{aligned}
$$

where, $\left\langle|\Delta \vec{r}(t)|^{2}\right\rangle$ is the MSD of oxygen atom of water.

Figure 4 depicts simulated $X_{\text {Solute }}$ dependent RMSDs $\left\lfloor\left\langle|\Delta \phi(t)|^{2}\right\rangle\right\rfloor$ (upper panels) and MSDs $\left\lfloor\left\langle|\Delta r(t)|^{2}\right\rangle\right\rfloor$ (lower panels) of water molecules in these two binary mixtures, TMAO/water and TMU/water. Here, we have used log-log scale for an expanded view of the short time ( $t^{2}$ dependence) and long time ( $t^{1}$ dependency) dynamical behaviours of RMSDs and MSDs. The ratio between $D_{R}$ and $D_{T}$ of water molecules, $D_{R} / D_{T}$,

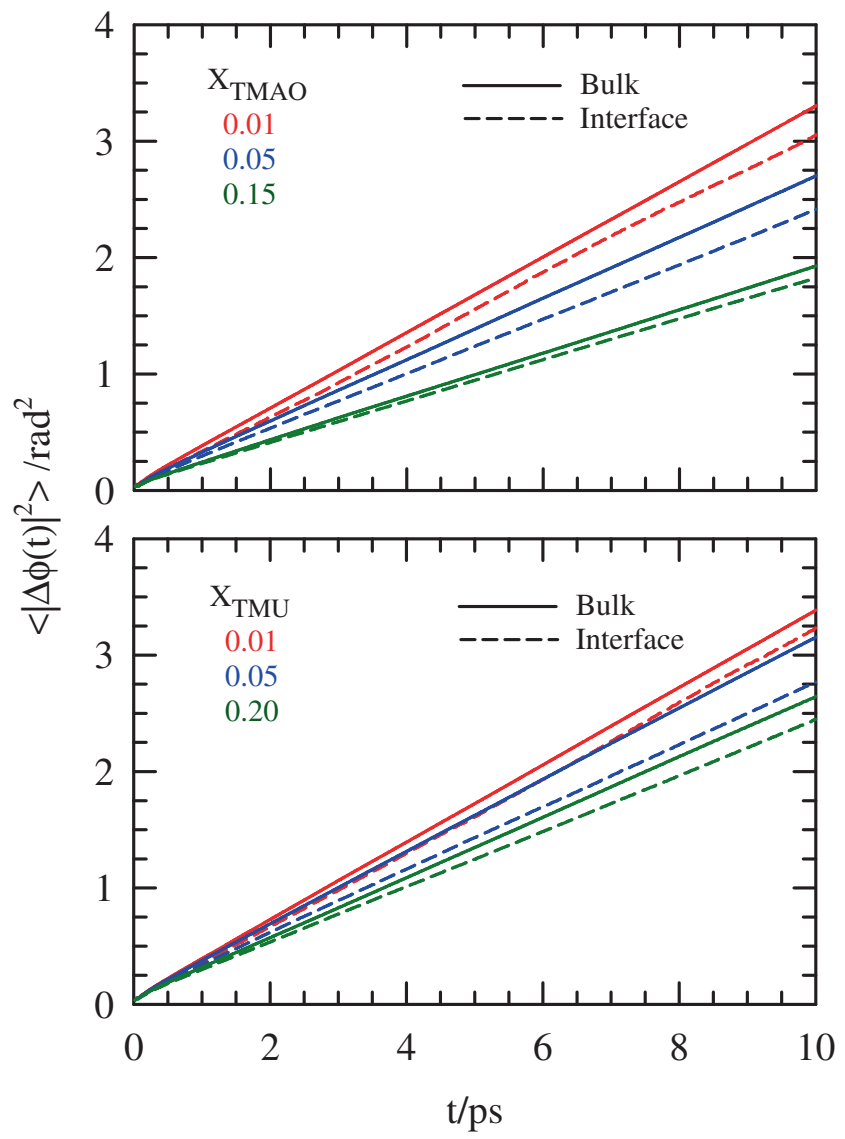

Figure 6. Plot of rotational mean squared displacement (RMSD), $\left\langle|\Delta \phi(t)|^{2}\right\rangle$ with time, $t$ for bulk (solid lines) and interfacial (dashed lines) water $-\mathrm{OH}$ bonds at three different $X_{\text {Solute }}$. Representations are color coded. 
calculated from the slopes of these simulated RMSDs and MSDs, respectively, are presented in the upper panel of Figure 5. Hydrodynamic predictions ${ }^{37}$ for the ratio, $D_{R} / D_{T}$, using the simulated viscosity coefficients $(\eta)$ and stick boundary condition (assuming water being spherical, $D_{R} / D_{T}=3 / 4 r^{2}, r$ being the diffuser radius) are also shown in the same panel for a comparison.

Note the deviation from hydrodynamics is quite strong, particularly at large $X_{\text {Solute }}$. This insufficiency of hydrodynamics may imply aqueous dynamics being heterogeneous for these amphiphile solutions. Larger deviation from hydrodynamics at higher TMAO concentrations relative to that in the corresponding TMU/water solutions indicates that TMAO perturbs the water dynamics to a greater extent than TMU. Simulated $\eta$ using the method described earlier ${ }^{24}$ is compared with the experimental data ${ }^{51}$ in the lower panel. Clearly, the present simulations can reproduce only qualitatively the experimental $X_{\text {Solute }}$ dependence of $\eta$ for these solutions. This, however, does not affect the hydrodynamic predictions for the $X_{\text {Solute }}$ dependent $D_{R} / D_{T}$ as $\eta$ cancels out.

Next, we simulate RMSDs and MSDs for those water molecules which reside within $5 \AA$ radius of a solute (TMAO or TMU) at initial time in these solutions at different solute concentrations. This is done in order to explore the extent of slowing down for interfacial water molecules. Various RDFs simulated for these water molecules indicate $\sim 20-25$ water molecules within such a shell which agrees well with the experimentally estimated ${ }^{11}$ total number of water molecules in the first solvation shells of these solutes. Figure 6 shows the $X_{\text {Solute }}$ dependent RMSDs for interfacial and bulk water molecules in these solutions.

As rotation is faster than translation, $D_{R}$ for interfacial water has been calculated within $10 \mathrm{ps}$ time window, whereas, $D_{T}$ for the same has been calculated

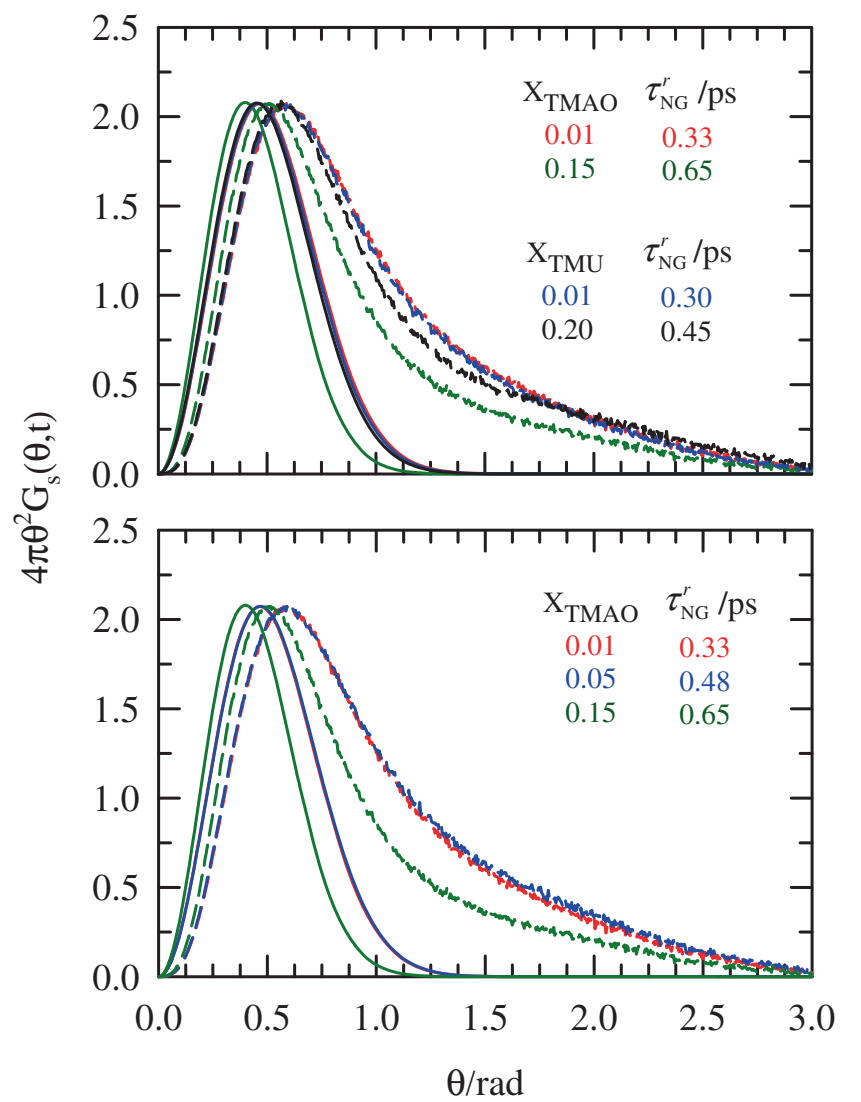

Figure 7. Angular distribution of time-dependent rotational van Hove self correlation function, $G_{S}(\theta, t)$ for water $-\mathrm{OH}$ bond at the lowest and the highest mole fractions of TMAO and TMU (upper panel) studied here and at different TMAO concentrations (lower panel). Dashed lines represent simulated distributions and the solid lines depict the corresponding Gaussian distribution. The times at which these distributions were determined are shown in both the panels. Representations are color-coded. Notice simulations suggest substantial amount of large angle displacements (>60 degree) for water molecules in these solutions which are absent in the calculated Gaussian distributions. $1 \mathrm{rad} \approx$ $57.29^{\circ}$.

Table 1. Rotational and translational diffusion coefficients $\left(D_{R}\right.$ and $\left.D_{T}\right)$ for bulk and interfacial water at different solute concentrations.

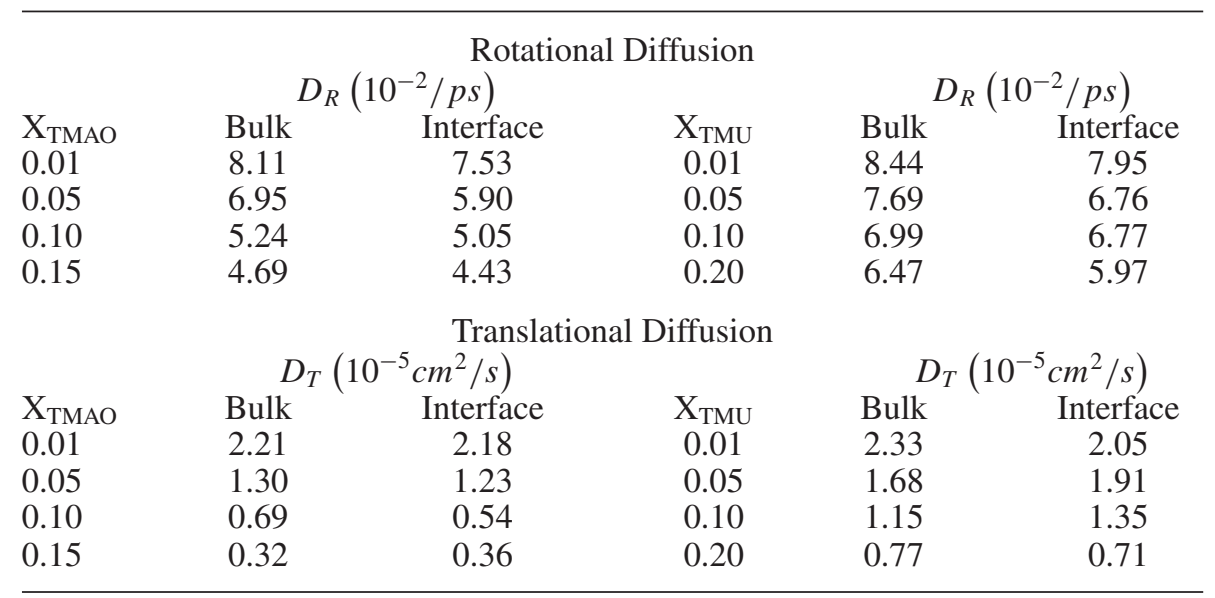


within $20 \mathrm{ps}$ time window. The translational MSDs are presented in Figure S3 (Supporting Information). Table 1 compares the interfacial and bulk diffusion coefficients for these solutes which indicate the interfacial effects being stronger for rotation than translation. These data therefore clearly indicate that water molecules which are at the interfaces diffuse at a somewhat slower rate than those in the bulk. This is in general agreement with earlier experimental ${ }^{11-17}$ and simulation $^{19,20}$ findings. However, the extent of slowing down found here is much less, the interfacial diffusion coefficients being always within $\sim 20 \%$ of those simulated for the bulk. As pointed out in a very recent work, ${ }^{23}$ inadequate representation of charge distributions in the water model used here can play a major role in producing such a small interfacial effects in the present simulations.

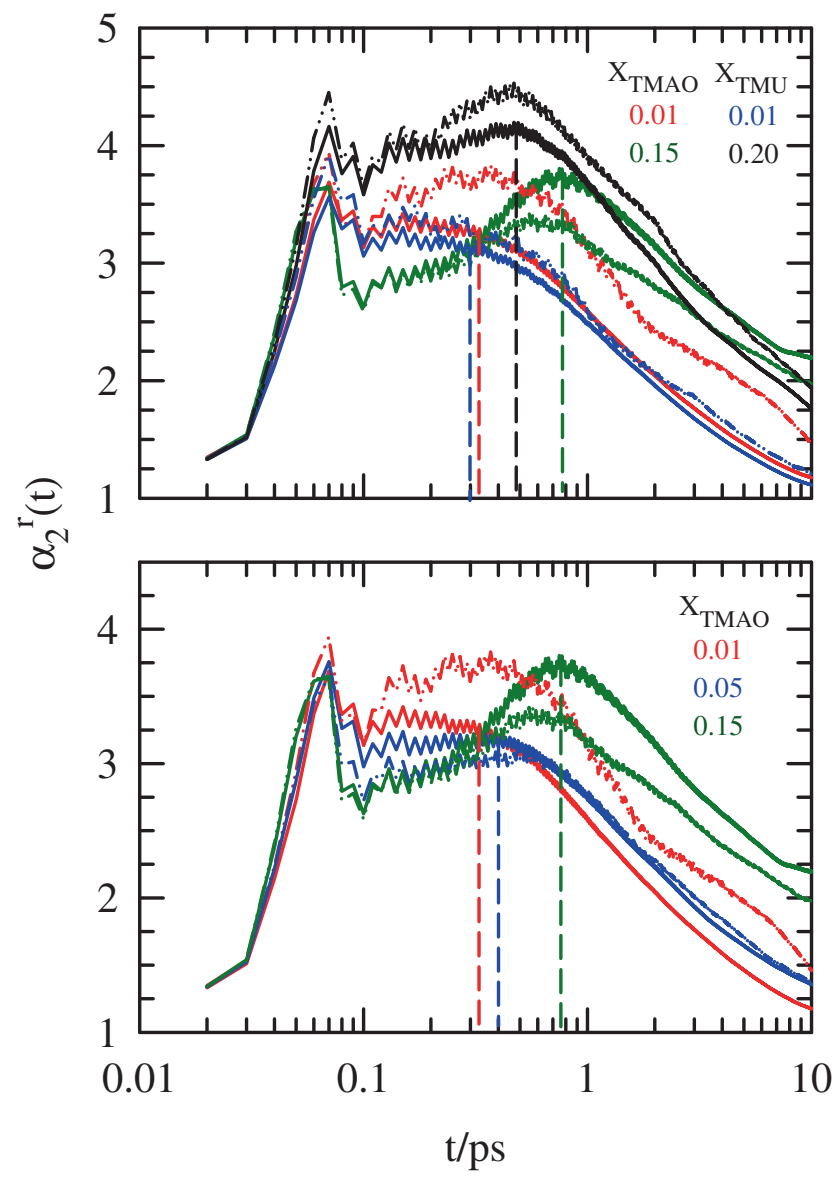

Figure 8. Time-dependent rotational non-Gaussian parameter, $\alpha_{2}^{r}(t)$, for bulk (solid lines) and interfacial (dash-dotdot lines) water $-\mathrm{OH}$ bonds at two different concentration regimes of TMAO and TMU (upper panel) and at various concentrations of TMAO (lower panel). Vertical dashed lines indicate peak positions of $\alpha_{2}^{r}(t)$ on time axis for bulk solutions. Representations are color-coded.

\subsection{Rotational and Translational Dynamic \\ Heterogeneity}

The time-dependent self part of the rotational van Hove correlation function $\left[G_{s}(\theta, t)\right]$ for angular displacements of $-\mathrm{OH}$ bond of water is shown in Figure 7. To

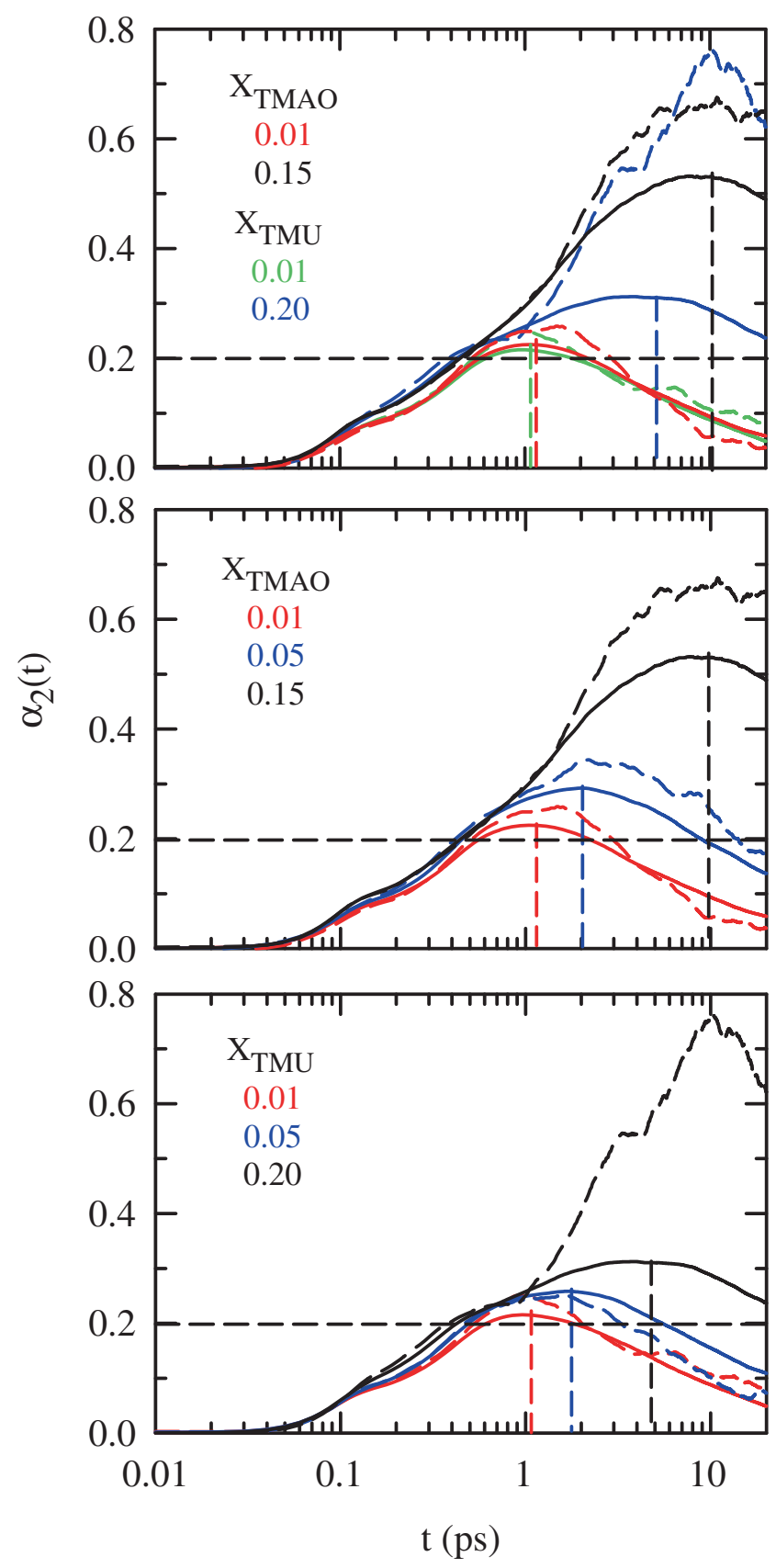

Figure 9. Distribution of translational non-Gaussian parameter, $\alpha_{2}(t)$ of bulk (solid lines) and interfacial (broken lines) water with time, $t$ at two different concentrations of TMAO and TMU (upper panel). Middle and lower panels show distribution of $\alpha_{2}(t)$ with $t$ at different concentrations of TMAO and TMU, respectively. Vertical dashed lines are drawn for each solute concentration to show the corresponding peak positions of $\alpha_{2}(t)$ for bulk water. 
compare the solute effect on the angular displacement of water, $\left[G_{s}(\theta, t)\right]$ at the lowest and the highest concentrations of both TMAO and TMU in water are shown in the upper panel of the figure. Composition dependent $\left[G_{s}(\theta, t)\right]$ are displayed in the lower panel of the figure for TMAO/water system and in Figure S4 (Supplementary Information) for TMU/water system respectively. Corresponding calculated Gaussian distributions are also shown in each panel. $G_{s}(\theta, t)$ has been calculated at times $\left[\tau_{N G}^{r}\right]$ when rotational NG parameter $\left[\alpha_{2}^{r}(t)\right]$ reaches the maximum. A comparison between the simulated and calculated distributions clearly indicates deviations from the Gaussian behaviour, suggesting heterogeneous distributions of angular displacements of water molecules in solutions of both TMAO and TMU.

A closer view of the dynamic heterogeneity (DH) of these solutions can be accessed by computing the $\mathrm{NG}^{33,52-54}$ and $\mathrm{NNG}^{34,52-54}$ parameters from these angular and centre-of-mass displacements. For example, the rotational NG parameter, $\alpha_{2}^{r}(t)$, obtained via the expression

$$
\alpha_{2}^{r}(t)=\frac{3}{5} \frac{\left\langle\Delta \phi^{4}(t)\right\rangle}{\left\langle\Delta \phi^{2}(t)\right\rangle^{2}}-1,
$$

with $\left\langle\Delta \phi^{2}(t)\right\rangle=\left\langle\left|\Delta \vec{\phi}_{i}(t)\right|^{2}\right\rangle$, and $\left\langle\Delta \phi^{4}(t)\right\rangle=$ $\left\langle\left|\Delta \vec{\phi}_{i}(t)\right|^{4}\right\rangle$, is presented in Figure 8 for interfacial (dash-dot-dot lines) and bulk (solid lines) water

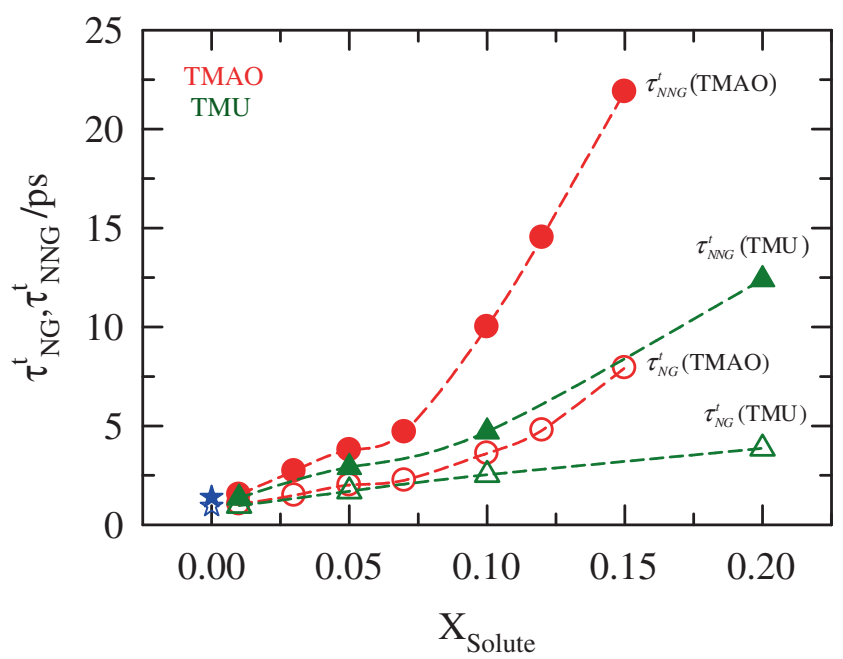

Figure 10. $X_{\text {Solute }}$ dependence for the peak-times of the translational non-Gaussian parameter $\left(\tau_{N G}^{t}\right)$ and the new non-Gaussian parameter $\left(\tau_{N N G}^{t}\right)$ for (water + TMAO) and (water + TMU) systems. Filled symbols represent $\tau_{N N G}^{t}$ and open symbols $\tau_{N G}^{t}$. Representations are color-coded. $\tau_{N G}^{t}$ and $\tau_{N N G}^{t}$ for ambient water are also shown by stars (blue). molecules. $\alpha_{2}^{r}(t)$ at two representative concentrations of TMAO and TMU into water are shown in the upper panel, whereas TMAO composition dependent $\alpha_{2}^{r}(t)$ are depicted in the lower panel.

TMU composition dependent $\alpha_{2}^{r}(t)$ is represented in Figure S5 (Supplementary Information). The nonmonotonic time dependence with a large non-zero peak value of $\alpha_{2}^{r}(t)$ suggests presence of strong DH in both the systems. A closer inspection of these curves reveals that the peak beyond $100 \mathrm{fs}$ of $\alpha_{2}^{r}(t)$ at different $X_{\text {Solute }}$ is occurring at a relatively longer time for TMAO than TMU. This may be due to the stronger water-TMAO interaction which subsequently imparts more heterogeneity in the water dynamics. $\alpha_{2}^{r}(t)$ peaks also shift to the longer times with increase of $X_{\text {Solute }}$.

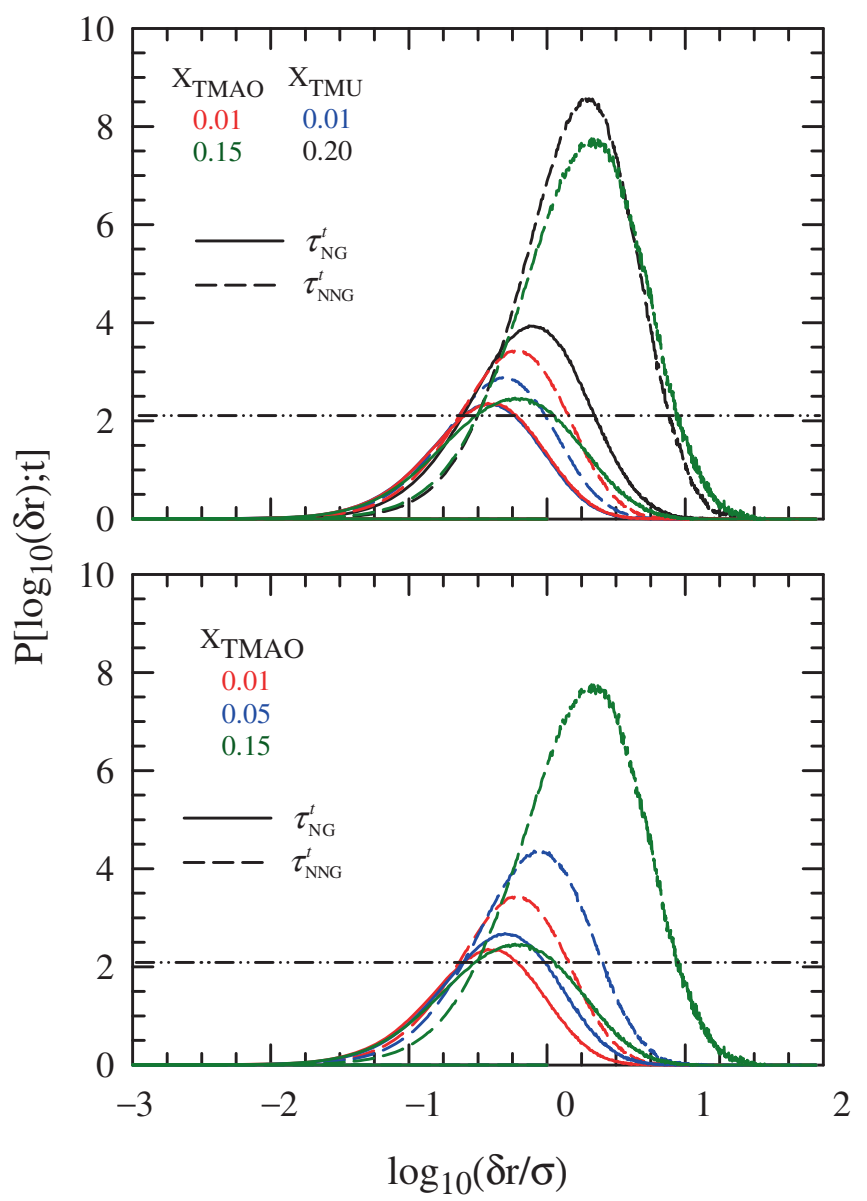

Figure 11. Comparison of $X_{\text {Solute }}$ dependence of the simulated single particle displacement distributions, $P\left[\log _{10}(\delta r) ; t\right]$ for water at two different concentrations of TMAO and TMU (upper panel) at the peak-times of NG (solid line) and NNG (dashed lines) parameters. TMAO concentration-dependent $P\left[\log _{10}(\delta r) ; t\right]$ are shown in the lower panel. Representations are, as before, color-coded. The height of the $P\left[\log _{10}(\delta r) ; t\right]$ corresponding to a Gaussian $G_{S}(\delta r, t)$ at these times is shown by horizontal broken lines. 

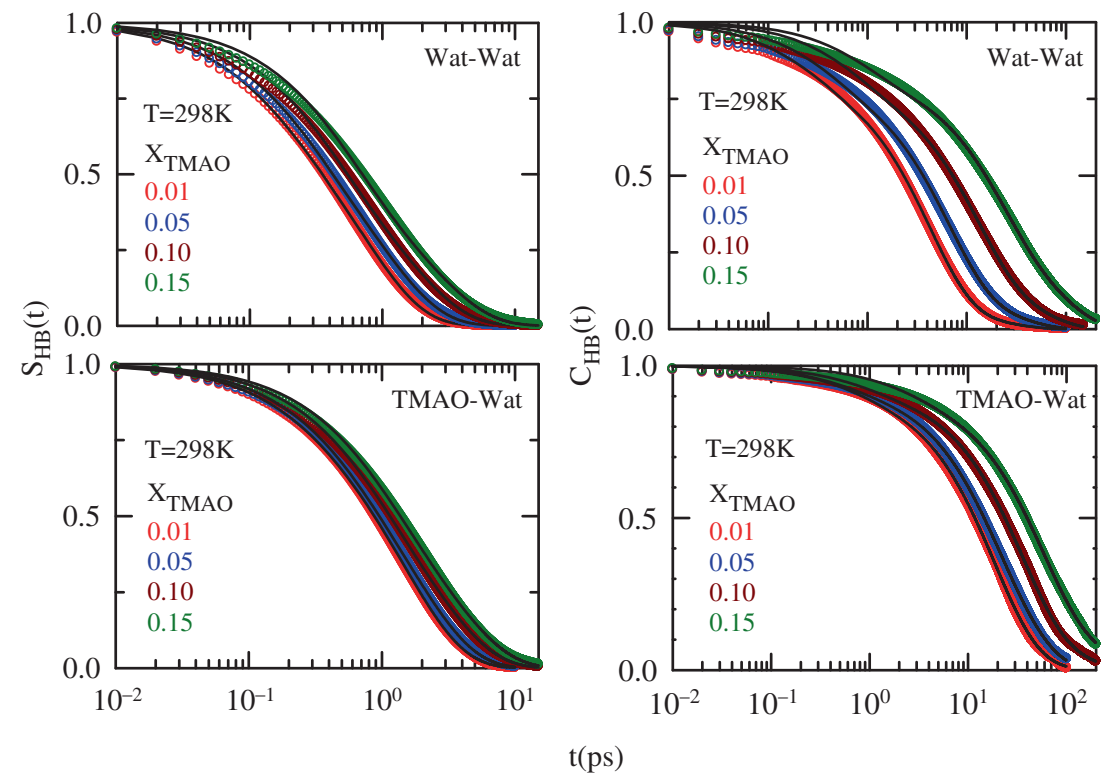

Figure 12. $X_{T M A O}$-dependent relaxations of $S_{H B}(t)$ and $C_{H B}(t)$ for waterwater hydrogen bonding (upper panel) and water-TMAO hydrogen bonding (lower panel). Simulated data are shown by the circles. Multi-exponential fits of the simulated data are shown by solid lines. $X_{T M A O}$ are color-coded as follows: 0.01: red, 0.05: blue, 0.10: dark red, 0.15: dark green.

Figure 9 depicts the corresponding translational NG parameter, $\alpha_{2}^{t}(t)$, which is obtained by using Eq. 4 after replacing $\left\langle\Delta \phi^{2}(t)\right\rangle$ and $\left\langle\Delta \phi^{4}(t)\right\rangle$ by $\left\langle\Delta r^{2}(t)\right\rangle$ and $\left\langle\Delta r^{4}(t)\right\rangle$, respectively. Similar non-monotonic dependence has been observed at all the $X_{\text {Solute }}$ considered here, with peak times $\left(\tau_{N G}^{t}\right)$ occurring at longer times for TMAO solutions than for TMU solutions. Also $\tau_{N G}^{t}$ appears at the longer timescales with increase of $X_{\text {Solute }}$. This suggests that the $X_{\text {Solute }}$ dependent DH timescales in TMAO solutions are longer than those in TMU solutions. Interestingly, $\alpha_{2}^{t}(t)$ peak heights for TMAO solutions are uniformly larger at all these $X_{\text {Solute }}$ than for TMU solutions, reflecting more heterogeneous water dynamics in presence of TMAO. For further characterization of $\mathrm{DH}$, we have calculated the translational NNG parameter, $\gamma(t)$, for these solutions at various $X_{\text {Solute }}$ by using the following expression ${ }^{34}$ from the simulated MSDs

$$
\gamma(t)=\frac{1}{3}\left\langle\Delta r^{2}(t)\right\rangle\left\langle\frac{1}{\Delta r^{2}(t)}\right\rangle-1,
$$

and shown in Figures S6 and S7 (Supplementary Information). As observed for $\alpha_{2}^{t}(t)$, here also the peak-time $\left(\tau_{N N G}^{t}\right)$ shifts to longer times with $X_{\text {Solute }}$, and also for changing the TMU solutions to TMAO solutions. Peaktimes slower than those in $\alpha_{2}^{t}(t)$ appears, suggesting that the $\mathrm{DH}$ can persist over a timescale longer than those reflected by $\tau_{N G}^{t}$.
Figure 10 presents a comparison between the $X_{\text {Solute }}$ dependent $\tau_{N G}^{t}$ and $\tau_{N N G}^{t}$ which indicates presence of similar DH timescales for both the systems at low solute concentrations. However, significantly longer peak-times characterize TMAO solutions at higher concentrations. Interestingly, these solution DH timescales increase with increase of $X_{\text {Solute }}$, and can even become approximately an order of magnitude longer than those obtained for neat ambient water. ${ }^{24}$

DH signatures for water molecules in these solutions can be further examined by investigating the probability distributions of logarithm of the single particle displacements at any given time, $P\left[\log _{10}(\delta r) ; t\right]$, which can be readily obtained from the self part of the van Hove correlation function, $G_{s}(\delta r, t)$, as follows: ${ }^{34,55}$

$$
P\left[\log _{10}(\delta r) ; t\right]=\ln (10) 4 \pi \delta r^{3} G_{s}(\delta r, t) .
$$

For a Gaussian $G_{s}(\delta r, t), P\left[\log _{10}(\delta r) ; t\right]$ becomes independent of time and attains a peak height of $\sim 2.13$. Figure 11 and Figure S8 (Supporting Information) depict the $X_{\text {Solute }}$ dependent $P\left[\log _{10}(\delta r) ; t\right]$ obtained from the corresponding simulated $G_{s}(\delta r, t)$ for water molecules in TMAO and TMU containing solutions. The deviation from Gaussian behaviour is quite evident for both the solutions and the extent of deviation increases upon increasing the solute concentration in the solution. 


\subsection{Hydrogen Bond Relaxation Dynamics}

It would be interesting to investigate the H-bond relaxation timescales in such dynamically heterogeneous environments where one of the specie (TMAO here) interacts more strongly than the other (TMU). The H-bond relaxation time has been calculated by two different correlation functions: H-bond lifetime relaxation $S_{H B}(t)$ and H-bond structural relaxation $C_{H B}(t)$ as follows: $:^{38-40}$

$$
S_{H B}(t)=\frac{\langle h(0) H(t)\rangle}{\langle h\rangle},
$$
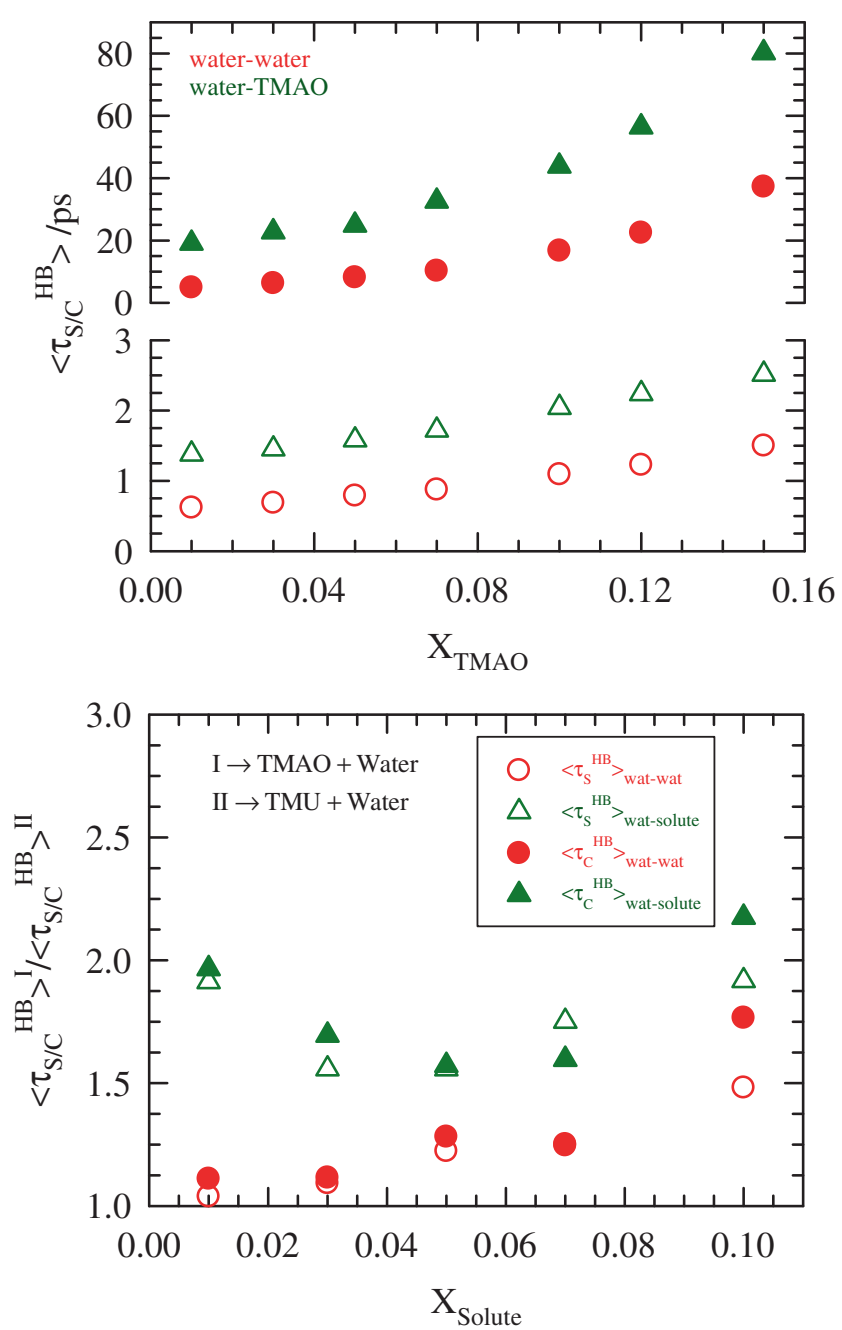

Figure 13. Plot of average times for H-bond lifetime and structural relaxation at different $X_{T M A O}$ (upper panel) and the ratio of average times obtained from $S_{H B}(t)$ and $C_{H B}(t)$ decay in both TMAO/Water and TMU/Water binary mixtures (lower panel). Average timescales from $S_{H B}(t)$ and $C_{H B}(t)$ decay are shown by open and filled symbols, respectively. Red circles and green triangles represent water-water and water-solute H-bonding, respectively.

$$
C_{H B}(t)=\frac{\langle h(0) h(t)\rangle}{\langle h\rangle} .
$$

Here, the operator $H(t)$ or $h(t)$ is unity when the tagged pair of molecules are H-bonded up to time $t$. The difference in calculation of these two correlation functions is that $C_{H B}(t)$ is independent of possible breaking of $\mathrm{H}$-bonds between the tagged pair in the intermediate time. Here, we have applied the same geometric criteria as used before. ${ }^{24}$ Figure 12 shows the decay of $S_{H B}(t)$ and $C_{H B}(t)$ for water-water and water-TMAO $\mathrm{H}$-bonding at various TMAO mole fractions along with the fitting. Bi-exponential and tri-exponential functions were required to fit these $S_{H B}(t)$ and $C_{H B}(t)$ decays respectively. Similar multi-exponential behaviour for these relaxation functions were also observed earlier for aqueous TMU solutions. ${ }^{24}$ Fitting parameters are summarized in Tables S9-S12 (Supplementary Information). In the upper panel of Figure 13, average relaxation times $\left\langle\tau_{S / C}^{H B}\right\rangle$ for water-water and water-TMAO $\mathrm{H}$-bonds at different $X_{T M A O}$ are shown. The ratio between the average $\mathrm{H}$-bond relaxation timescales at a given concentration for TMAO and TMU solutions, $\left\langle\tau_{S / C}^{H B}\right\rangle^{T M A O} /\left\langle\tau_{S / C}^{H B}\right\rangle^{T M U}$, for the common concentration range are presented in the lower panel of this figure. As observed before in TMU/water mixture, ${ }^{24}\left\langle\tau_{S / C}^{H B}\right\rangle$ for water-TMAO $\mathrm{H}$-bonding is longer than that for water-water $\mathrm{H}$-bonding in $\mathrm{TMAO} /$ water mixture, and the extent of lengthening increases with $X_{T M A O}$. The $X_{\text {Solute }}$ dependent ratio of average times shown in this figure is always greater than unity. This clearly indicates that $\mathrm{H}$-bonds in TMAO/water system are longerlived than in TMU/water system. Increase of this ratio with $X_{\text {Solute }}$ also suggests that TMAO has larger confining ability for water than TMU. More than two-fold lengthening of $\mathrm{H}$-bond fluctuation timescales have been observed on moving from TMU to TMAO solutions. Here, H-bond relaxation dynamics nicely correlates with the result that hydrophilic group of TMAO (the oxygen atom) interacts more strongly with water than the oxygen atom of TMU. This has also been the observation for various $\mathrm{DH}$ parameters investigated in this work.

\section{Conclusions}

In conclusion, $\mathrm{DH}$ in TMAO/water and TMU/water solutions were found to be moderately different although these two solutes are entirely different in their chemical nature and biological actions. The zwitterionic property of TMAO does induce enhanced interaction with water molecules compared to that observed for TMU but this has no bearing on the simulated DH 
timescales and single particle distributions at low solute concentrations. But in concentrated solutions, these two solutes show markedly different DH timescales. Interfacial diffusion coefficients have been found to be somewhat smaller than those for bulk solutions, agreeing qualitatively with the existing experimental and simulation results. The extent of slowing down is, however, small ( $\sim 20 \%$ at the most) which may be due either to the insufficient modelling of charge distributions for the SPC/E water or to the choice of interfacial region $(\sim 5 \AA)$ implemented here. A closer inspection of simulated $\mathrm{DH}$ parameters appears to reveal that aqueous TMAO solutions are somewhat more dynamically heterogeneous than the TMU counter-part. Simulated H-bond relaxation timescales have also been found to be longer-lived in TMAO solutions than in TMU solutions. Similar investigation on water/alcohol systems $^{56-59}$ along the line of what has already been done for aqueous solutions of ionic liquids ${ }^{60-62}$ would be useful to understand the impact of alcohol aggregation on water dynamics. But one needs to be cautious about the choice of the model potential because, for example, the model potential for flexible $\mathrm{TMAO}^{63}$ predicts faster dynamics than by the rigid model used here. A comparison of these results is shown in the Supplementary Information. Furthermore, DH analyses of non-aqueous ${ }^{64}$ and non-associative neat solvents, ${ }^{65}$ and amide deep eutectics ${ }^{66-68}$ are required to establish the generality of spatio-temporal heterogeneity in a variety of systems at ambient condition.

\section{Supplementary Information (SI)}

Supplementary Information contains figures of radial distribution functions between different atomic pairs in TMAO/water and TMU/water binary mixtures, rotational and translational mean squared displacements of both interfacial and bulk water in these binary mixtures, angular distribution of time-dependent rotational van Hove self correlation function rotational NG parameter and single particle displacement distribution for TMU/water system, translational NG and NNG for both the binary mixtures, water-water and water-TMAO H-bond lifetime and structural relaxation decays, etc. Tables are given for rotational and translational diffusion coefficients of both interfacial and bulk water, bi-exponential and tri-exponential fit parameters for $\mathrm{S}_{H B}(\mathrm{t})$ and $\mathrm{C}_{H B}(\mathrm{t})$ relaxations. Some results obtained by considering rigid and flexible models of TMAO are also shown to compare between these two models. Supplementary Information is available at www.ias.ac.in/ chemsci.

\section{Acknowledgements}

We gratefully acknowledge the TUE-CMS computational facility via the project, SR/NM/NS-29/2011(G) at the S. N. Bose Centre.

\section{References}

1. Burg M B and Ferraris J D 2008 J. Biol. Chem. 2837309

2. Kempf B and Bremer E 1998 Arch. Microbiol. 170319

3. Yancey P H 2001 Am. Zool. 41699

4. Daggett V 2006 Chem. Rev. 1061898

5. Pace C N and Marshall H F 1980 Arch. Biochem. Biophys. 199270

6. Barone G, del Vecchio P, Giancola C and Graziano G G 1993 Thermochim. Acta 22767

7. Klimov D K, Straub J E and Thirumalai D 2004 Proc. Natl. Acad. Sci. U.S.A. 10114760

8. Mountain R D and Thirumalai D 2003 J. Am. Chem. Soc. 1251950

9. Cser L, Jancsó G, Papoular R and Grósz T 1989 Physica $B$ 156\&157 145

10. Doi H, Watanabe Y and Aida M 2014 Chem. Lett. 43 865

11. Mazur K, Heisler I A and Meech S R 2011 J. Phys. Chem. B 1152563

12. Hunger J, Tielrooij K, Buchner R, Bonn M and Bakker H J 2012 J. Phys. Chem. B 1164783

13. Tielrooij K, Hunger J, Buchner R, Bonn M and Bakker H J 2010 J. Am. Chem. Soc. 13215671

14. Rezus Y L A and Bakker H J 2007 Phys. Rev. Lett. 99 148301

15. Rezus Y L A and Bakker H J 2009 J. Phys. Chem. B 113 4038

16. Rezus Y L A and Bakker H J 2008 J. Phys. Chem. A 112 2355

17. Petersen C, Bakulin A A, Pavelyev V G, Pshenichnikov M S and Bakker H J 2010 J. Chem. Phys. 133164514

18. Paul S and Patey G N 2006 J. Phys. Chem. B 11010514

19. Laage D, Stirnemann G and Hynes J T 2009 J. Phys. Chem. B 1132428

20. Stirnemann G, Sterpone F and Laage D 2011 J. Phys. Chem. B 1153254

21. Almásy L, Len A, Székely N K and Pleštil J 2007 Fluid Phase Equilib. 257114

22. Shikata T and Itatani S 2002 J. Solution Chem. 31823

23. Usui K, Hunger J, Sulpizi M, Ohto T, Bonn M and Nagata Y 2015 J. Phys. Chem. B 11910597

24. Indra S and Biswas R 2015 Mol. Sim. 41471

25. Titantah J T and Karttunen M 2012 J. Am. Chem. Soc. 1349362

26. Frank H S and Evans M W 1945 J. Chem. Phys. 13507

27. Marcus Y 2002 Phys. Chem. Chem. Phys. 44462

28. Fornili A, Civera M, Sironi M and Fornili S L 2003 Phys. Chem. Chem. Phys. 54905

29. Qvist J and Halle B 2008 J. Am. Chem. Soc. 13010345

30. Imoto S, Forbert H and Marx D 2015 Phys. Chem. Chem. Phys. 1724224

31. Brandeburgo W H, van der Post S T, Meijerab E J and Ensing B 2015 Phys. Chem. Chem. Phys. 1724968

32. Knake L, Schwaab G, Kartaschew $K$ and Havenith $M$ 2015 J. Phys. Chem. B 11913842 
33. Rahman A 1964 Phys. Rev. 136405

34. Flenner E and Szamel G 2005 Phys. Rev. E 72 011205

35. Mazza M G, Giovambattista N, Starr F W and Stanley H E 2006 Phys. Rev. Lett. 96057803

36. Lombardo T G, Debenedetti P G and Stillinger F H 2006 J. Chem. Phys. 125174507

37. Hansen J P and McDonald I R 1986 In Theory of Simple Liquids (New York: Academic Press)

38. Luzar A 1996 Faraday Discuss. 10329

39. Chandra A 2000 Phys. Rev. Lett. 85768

40. Balasubramanian S, Pal S and Bagchi B 2002 Phys. Rev. Lett. 89115501

41. Berendsen H J C, Grigera J R and Straatsma T P 1987 J. Phys. Chem. 916269

42. Allen M P and Tildesley D J 1987 In Computer Simulations of Liquids (New York: Oxford University Press)

43. Martinez L, Andrade R, Birgin E G and Martinez J M 2009 J. Comput. Chem. 302157

44. Smith W, Forester T R and Todorov I T, DL_POLY Classic (Daresbury: STFC Daresbury Laboratory) (2012)

45. Okpala C, Guiseppi-Elie A and Maharajh D M $1960 \mathrm{~J}$. Chem. Eng. Data 25384

46. Zou Q, Bennion B J, Daggett V and Murphy P $2002 \mathrm{~J}$. Am. Chem. Soc. 1241192

47. Nose S 1984 J. Chem. Phys. 81511

48. Hoover W G 1985 Phys. Rev. A 311695

49. Ryckaert J P, Ciccotti G and Berendsen H J C 1977 J. Comput. Phys. 23327

50. Kämmerer S, Kob W and Schilling R 1997 Phys. Rev. E 565450
51. Sinibaldi R, Casieri C, Melchionna S, Onori G, Segre A L, Viel S, Mannina L and De Luca F 2006 J. Phys. Chem. B 1108885

52. Pal T and Biswas R 2013 Theor. Chem. Acc. 1321348

53. Indra S, Guchhait B and Biswas R 2016 J. Chem. Phys. 144124506

54. Das A, Das S and Biswas R 2015 J. Chem. Phys. 142 034505

55. Pal T and Biswas R 2014 J. Chem. Phys. 141104501

56. Indra S and Biswas R 2015 J. Chem. Phys. 142204501

57. Pradhan T, Ghoshal P and Biswas R 2008 J. Phys. Chem. A 112915

58. Pradhan T, Ghoshal P and Biswas R 2008 J. Chem. Sci. 120275

59. Gazi H A R and Biswas R 2011 J. Phys. Chem. A 115 2447

60. Pal T and Biswas R 2015 J. Phys. Chem. B 11915683

61. Daschakraborty S and Biswas R 2016 J. Chem. Phys. 144104505

62. Daschakraborty S and Biswas R 2014 J. Chem. Phys. 140014504

63. Kast K M, Brickmann J, Kast S M and Berry R S 2003 J. Phys. Chem. A 1075342

64. Kashyap H K, Pradhan T and Biswas R 2006 J. Chem. Phys. 125174506

65. Biswas R and Bagchi B 1999 J. Phys. Chem. A 1032495

66. Guchhait B, Gazi H A R, Kashyap H K and Biswas R 2010 J. Phys. Chem. B 1145066

67. Guchhait B, Daschakraborty S and Biswas R $2012 \mathrm{~J}$. Chem. Phys. 136174503

68. Gazi H A R, Guchhait B, Daschakraborty S and Biswas R 2011 Chem. Phys. Lett. 501358 